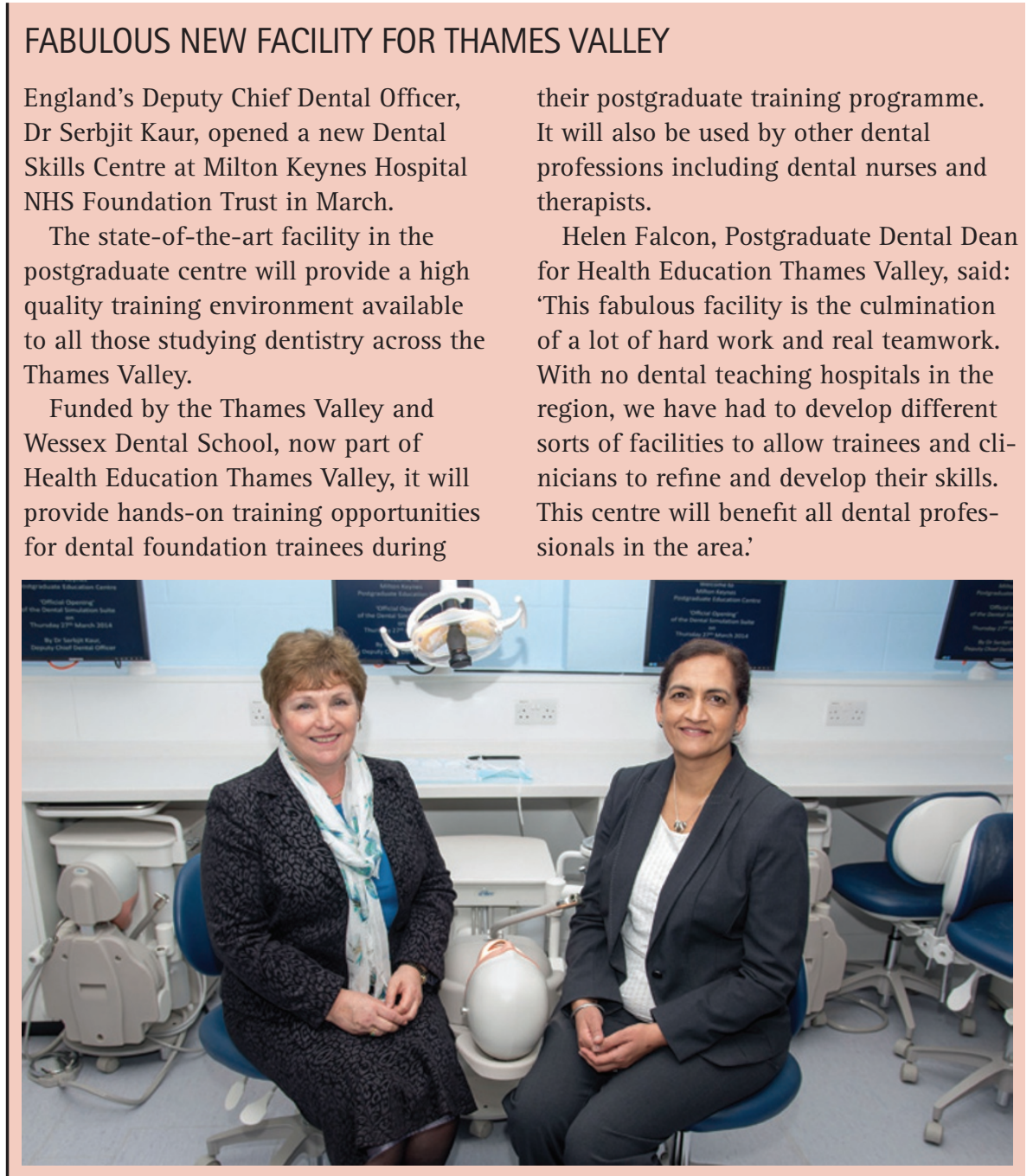

\section{PANDAS LOVE SWEETIES TOO}

Despite their bamboo-chomping diet, giant pandas possess functional sweet taste receptors and in tests, show a strong preference for some natural sweeteners, including fructose and sucrose.

Researchers at the Monell Chemical Senses Center in Philadelphia studied giant pandas as part of a long-term project focused on understanding how taste preferences and diet selection are shaped by taste receptor genes. ${ }^{1}$

A previous study found that cats, which must eat meat in order to survive, had lost the ability to taste sweets due to a genetic defect that deac-

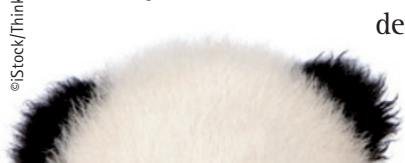
tivates the sweet taste receptor. Although giant pandas and cats belong to the same taxonomic order,
Carnivora, the giant pandas have a very different diet, as they feed almost exclusively on bamboo, a grass-like plant containing very small amounts of sugar. The researchers wondered whether giant pandas had also lost sweet taste perception, like cats.

Eight giant pandas in China were given two bowls of liquid, one containing water and one a solution of water mixed with one of six different natural sugars. All of the pandas preferred the sugar solutions to plain water, and this was especially evident for fructose and sucrose.

Using DNA collected from the giant pandas during routine health examinations, genes that code for the panda sweet taste receptor were isolated and then inserted into human host cells grown in culture. These cells responded vigorously to sugars; this step helped investigators confirm that pandas have a functional sweet taste receptor that underlies their ability to detect and respond to sugars.

1. Jiang $P$, Josue-Almqvist J, Li $X$ et al. The bambooeating giant panda (Ailuropoda melanoleuca) has a sweet tooth: behavioral and molecular responses to compounds that taste sweet to humans. PLoS One 2014; 9: e93043. DOI: 10.1371/journal. pone.0093043. 